\title{
Pulmonary nodules and mini-invasive lung resection: do we have the right "tool" for their intraoperative localization?
}

\author{
Paola Ciriaco, Piergiorgio Muriana, Giampiero Negri \\ Department of Thoracic Surgery, Scientific Institute and University Vita-Salute San Raffaele, Ospedale San Raffaele, Milan, Italy \\ Correspondence to: Paola Ciriaco, MD. Department of Thoracic Surgery, Scientific Institute and University Vita-Salute San Raffaele, Ospedale San \\ Raffaele, Via Olgettina 60, Milano 20132, Italy. Email: ciriaco.paola@hsr.it. \\ Provenance: This is an Invited Editorial commissioned by Section Editor Dr. Min Zhang (Department of Thoracic Oncology, The First Affiliated \\ Hospital of Chongqing Medical University, Chongqing, China). \\ Comment on: Kato H, Oizumi H, Suzuki J, et al. Thoracoscopic anatomical lung segmentectomy using 3D computed tomography simulation without \\ tumour markings for non-palpable and non-visualized small lung nodules. Interact Cardiovasc Thorac Surg 2017;25:434-41.
}

Submitted Sep 20, 2017. Accepted for publication Oct 05, 2017.

doi: $10.21037 /$ jtd.2017.10.87

View this article at: http://dx.doi.org/10.21037/jtd.2017.10.87

Lung resection still remains the best cure for early stage non-small cell lung cancer (NSCLC). Therefore, identification of the cancer in the early stage becomes the main goal, whenever a suspicious nodule is detected at chest CT scan.

The use of mini-invasive techniques, such as video thoracoscopy and robotic lung resection, is nowadays increasing in the thoracic surgery practice compared to the standard thoracotomy approach, changing in the last decade from $1.3 \%$ of VATS among all lobectomies to $13 \%$ (1). The escalation of mini-invasive techniques is connected with a significant reduced postoperative pain, lower morbidity, better preservation of pulmonary function and decrease of hospitalization associated with a better cosmetic result (2).

Pulmonary nodules (PNs) represent the most common reason for VATS and/robotic resections. Most lesions can be easily detected by inspection and tactile evaluation with an instrumental probe. However, some lung nodules are difficult to identify thoracoscopically. The small size of the nodule and/or their distance from the pleural surface might be limiting factors for a successful thoracoscopic resection (3). Moreover, ground glass opacities (GGOs) are more often detected at CT scan and they are challenging to be identified in the lung parenchyma even during a standard thoracotomy. A limited resection of GGO is nowadays the suggested procedure for their treatment, so a detailed localization of the lesion becomes more and more important in the surgical practice of lung resection (4).

Techniques to localize PNs vary from preoperative injection of methylene blue (5) or colored collagen (6) at the site of the PN to intraoperative ultrasound detection and CT-guided positioning of a metal wire (7-9). A failure rate of around $13 \%$ for methylene blue injection has been reported due to either an excess of liquid injected or an error in nodule localization (5). Intraoperative ultrasound detection requires a special flexible probe and it can be limited by the presence of air in the lung when complete collapse is not feasible (9). Moreover, despite the lack of complications and the high sensitivity and specificity found with the use of ultrasound (10) only a few cases are reported in the literature and it is known to present limitations in localizing inflammatory nodules (11). Probe and/or digital palpation could also be useful methods for localizing PNs, although these techniques are effective only when the nodule is superficial or $>20 \mathrm{~mm}$ in size. Microcoil and fiducial marker placement decreases the discomfort of patients during the waiting time to enter the operative room, compared to other invasive procedures, but requires fluoroscopic guidance during the surgical procedure, increasing radiation exposure for surgeons. The success rate is reported to be $93-98.4 \%$ with complications occurring in $3-10 \%$ of the patients including migration of the coil, air embolism and hemothorax (12). We have previously reported our experience with preoperative computed tomography-guided hook wire localization and 
we have found that it represents a useful marker with minor complications and a high rate of success in expert hands (3).

Most of the above described techniques are performed preoperatively in the radiology unit leading to a possible increase of the possibility of dislocation of the marker and/ or of the occurring of pneumothorax and hemothorax. The use of a hybrid operating room for the intraoperative assessment and localization of PNs is advocated by some authors $(13,14)$ although specific institutional organization is required for such procedure.

Despite the several possibilities of PN's localization, chest CT scan still remains the main technique to rely on and some Authors state the possibility to avoid markers positioning for $\mathrm{PN}$ with the use of three dimensional (3D) computed tomography simulation $(15,16)$. The development of multi-detector computed tomography has enabled 3D images of lung structures. The $3 \mathrm{D}$ reconstruction can be very helpful in anatomical lung resection, including segmentectomy which is a more complicated operative procedure than standard lobectomy for his anatomical complexity due to the variability of vascular and bronchial structures at different levels.

Kato and colleagues have reported an interesting series about thoracoscopic anatomical lung segmentectomy using 3D computed tomography simulation without tumour marking for PNs that were non-palpable and non-visualized (17). A detailed anatomical 3D pulmonary arteriovenous reconstruction was performed using a volume-rendering method and arteries and veins were distinguished interpreting the differences in contrast agent densities. A targeted segment on the CT image was identified containing the $\mathrm{PN}$ in the horizontal, coronal and sagittal planes, then the artery and the veins were recognized on the $3 \mathrm{D}$ image and finally measurements to obtain an adequate surgical margin were performed. $3 \mathrm{D}$ images were useful both before and during operation. The correct resection rate of the otherwise undetectable tumor was $100 \%(17)$.

Surely 3D CT simulation might represent a valid alternative to marker positioning for non-detectable $\mathrm{PNs}$, especially for intraparenchymal lesions requiring segmentectomy. This technique is safe and does not add complications to the surgical procedure. Therefore, it is important to make some considerations correlated first of all with the necessity of well trained personnel and secondary with the possible difference in measurements between an inflated lung at the CT scan and a collapsed one during surgery, leading to the need to expand the resection in order to obtain adequate surgical margins. Moreover, superiority of anatomical resections over wedge resection for low-grade lung tumor is still controversial in literature $(4,18)$.

In conclusion, the widespread performing of minimally invasive lung resections for early stage NSCLC has led to the need of a more accurate preoperative localization of PNs. Different techniques present advantages and complications that have to be evaluated according each Department experience. A close cooperation between thoracic surgeons and radiologists is often advocated for a successful procedure.

\section{Acknowledgements}

None.

\section{Footnote}

Conflicts of Interest: The authors have no conflicts of interest to declare.

\section{References}

1. Pages PB, Hanna HA, Bertaux AC, et al. Medicoeconomic analysis of lobectomy using thoracoscopy versus thoracotomy for lung cancer: a study protocol for a multicentre randomised controlled trial (Lungsc01). BMJ Open 2017;7:e012963.

2. Yan TD, Cao C, D'Amico TA, et al. Video-assisted thoracoscopic surgery lobectomy at 20 years: a consensus statement. Eur J Cardiothorac Surg 2014;45:633-9.

3. Ciriaco P, Negri G, Puglisi A, et al. Video-assisted thoracoscopic surgery for pulmonary nodules: rationale for preoperative computed tomography-guided hookwire localization. Eur J Cardiothorac Surg 2004;25:429-33.

4. Tsutani Y, Miyata Y, Nakayama H, et al. Appropriate sublobar resection choice for ground glass opacitydominant clinical stage IA lung adenocarcinoma. Chest 2014;145:66-71.

5. Kerrigan DC, Spence PA, Crittenden MD, et al. Methylene blue guidance for simplified resection of a lung lesion. Ann Thorac Surg 1992;53:163-4.

6. Nomori H, Horio H. Colored collagen is a long-lasting point marker for small pulmonary nodules in thoracoscopic operations. Ann Thorac Surg 1996;61:1070-3.

7. Mack MJ, Shennib H, Landreneu RJ, et al. Techniques for localization of pulmonary nodules for thoracoscopic resection. J Thorac Cardiovasc Surg 1993;106:550-3. 
8. Templiton P, Krasna N. Needle/wire lung nodule localization for thoracoscopic resection. Chest 1993;104:953-4.

9. Santambrogio R, Montorsi M, Bianchi P, et al. Intraoperative ultrasound during thoracoscopic procedures for solitary pulmonary nodules. Ann Thorac Surg 1999;68:218-22.

10. Tatsumura T. Preoperative and intraoperative ultrasonographic examination as an aid in lung cancer operations. J Thorac Cardiovasc Surg 1995;110:606-12.

11. Hida Y, Kato H, Nishibe T, et al. Value of intraoperative intrathoracic ultrasonography during video-assisted thoracoscopic pulmonary resection. Surg Laparosc Endosc 1996;6:472-5.

12. Lin MW, Chen JS. Image-guided techniques for localizing pulmonary nodules in thoracoscopic surgery. $J$ Thorac Dis 2016;8:S749-55.

13. Hsieh CP, Hsieh MJ, Fang HY, et al. Imaging-guided thoracoscopic resection of a ground-glass opacity lesion in a hybrid operating room equipped with a robotic C-arm CT system. J Thorac Dis 2017;9:E416-9.

Cite this article as: Ciriaco P, Muriana P, Negri G. Pulmonary nodules and mini-invasive lung resection: do we have the right "tool" for their intraoperative localization? J Thorac Dis 2017;9(11):4216-4218. doi: 10.21037/jtd.2017.10.87
14. Zhao ZR, Lau RW, Ng CS. Hybrid theatre and alternative localization techniques in conventional and single-port video-assisted thoracoscopic surgery. J Thorac Dis 2016; 8:S319-27.

15. Ikeda N, Yoshimura A, Hagiwara M, et al. Three dimensional computed tomography lung modeling is useful in simulation and navigation of lung cancer surgery. Ann Thorac Cardiovasc Surg 2013;19:1-5.

16. Mochizuki K, Tukatsuki M, Soyama A, et al. The usefulness of a high-speed 3D-image analysis system in pediatric living donor liver transplantation. Ann Transplant 2012;17:31-4.

17. Kato H, Oizumi H, Suzuki J, et al. Thoracoscopic anatomical lung segmentectomy using 3D computed tomography simulation without tumour markings for nonpalpable and non-visualized small lung nodules. Interact Cardiovasc Thorac Surg 2017;25:434-41.

18. Aberle DR, Adams AM, Berg CD, et al. Reduced lungcancer mortality with low-dose computed tomographic screening. N Engl J Med 2011;365:395-409. 